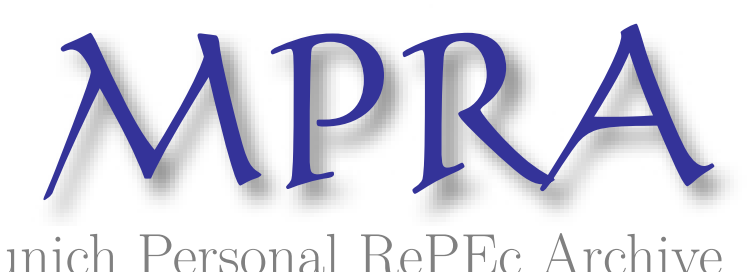

Munich Personal RePEc Archive

\title{
BUDGET POLICY AND ECONOMIC GROWTH IN RUSSIA. OPTIMAL BUDGET RULE
}

Skrypnik, Dmitriy

1 January 2016

Online at https://mpra.ub.uni-muenchen.de/75853/

MPRA Paper No. 75853, posted 06 Jul 2019 13:42 UTC 


\title{
БЮДЖЕТНАЯ ПОЛИТИКА И ЭКОНОМИЧЕСКИЙ РОСТ В РОССИИ. ОПТИМАЛЬНОЕ БЮДЖЕТНОЕ ПРАВИЛО
}

\begin{abstract}
Аннотация
В работе на основе макроэкономической модели российской экономики анализируется бюджетная политика в России в период бурного роста на нефть 2000-х. Новизна работы состоит в том, что в ней показана неоптимальность бюджетной политики в соответствующий период - фактический уровень государственных расходов в стране был ниже оптимального. Основной механизм возникновения роста в оптимальном сценарии связан с масштабирующим эффектом государственных расходов, повышающим производственные возможности экономики. Адекватная монетарная политика позволяет не допустить раскручивания инфляционной спирали и запустить спираль экономического роста. Неоптимальность бюджетной политики является следствием особенности механизма бюджетного правила, не учитывающего влияние государственных расходов на экономический рост, что соответствует типу управления по разомкнутому контуру. Бюджетное правило, реализующее управление по замкнутому контуру и позволяющее конструировать оптимальную экономическую политику для развивающихся стран, может стать основой формирования системы управления ростом, сочетающей универсальное и программное планирование. Ключевым принципом подобного правила должен стать принцип «нащупывания» экономического равновесия.
\end{abstract}

Ключевые слова: Оптимальное управление, макроэкономическая модель, бюджетное правило.

JEL коды: E620, O230, H540, C510, C520, C320. 


\title{
BUDGET POLICY AND ECONOMIC GROWTH IN RUSSIA. OPTIMAL BUDGET RULE
}

\author{
Candidate of Science (Economy) Skrypnik D.V.
}

\begin{abstract}
The article shows that actual public expenditure in the period of rapid oil prices growth of the 2000s was less than optimal level in Russia. The macroeconomic model of Russian economy is the basis of current research. The main mechanism of growth in an optimum scenario is associated with the scaling effect of public expenditure, which increases production possibilities of an economy. Adequate monetary policy allows to prevent unwinding of the inflation spiral and runs the growth spiral. Non-optimality of fiscal policy is a consequence of budget rule mechanism features, which do not take into account the influence of government expenditures on economic growth. The fiscal rule that implements the «closed loop» control and allows to design optimal economic policies for developing countries can become a basis for the system of growth management that combines universal and program planning. The key principle of optimal budget rule must be "tâtonnement" like process of budget parameters choice.
\end{abstract}

Keywords: optimal control, macroeconomic model, the fiscal rule.

JEL classification: E620, O230, H540, C510, C520, C320. 


\section{ВВЕДЕНИЕ ${ }^{1}$}

Центральным приоритетом экономической политики в России в период бурного роста цен на нефть 2000-х гг. стала макроэкономическая стабильность. Для достижения этой цели был предпринят ряд попыток встраивания механизма защиты от внешних шоков в бюджетную политику. Механизмы такого типа принято относить к бюджетным правилам.

Проводимая в России бюджетная политика часто становилась объектом дискуссии. Находились как сторонники полного отказа от механизма государственных (стабилизационных) фондов и бюджетного правила, так и сторонники еще более сдерживающей политики. Профессиональная дискуссия концентрировалась на вопросе обоснованности величины изъятия ликвидности мерами бюджетной политики. Один из доводов в пользу большего изъятия состоял в ограниченности спроса на деньги и возможном раскручивании инфляционной спирали в результате проведения менее рестриктивной бюджетной политики. С другой стороны, спрос на деньги возрастает в результате роста объема производства. Многообразие эффектов, возникающих в результате увеличения или снижения государственных расходов, однако, шире эффектов, связанных с денежным рынком. Кроме того, возникающие эффекты могут влиять и на сам спрос на деньги. Для анализа последствий изменения бюджетных расходов, таким образом, требуется более общая постановка задачи, предполагающая адекватное описание основных экономических механизмов системы и механизмов экономической политики.

Целью настоящей работы является определение оптимальной величины государственных расходов, позволяющее ответить на вопрос, был ли реализован потенциал бюджетной политики механизмом действующего в России бюд-

1 Работа была подготовлена под научным руководством академика РАН, д.э.н. В.М. Полтеровича, которому автор признателен за участие и плодотворные обсуждения. Автор выражает благодарность д.э.Н., профессору М.Ю. Афанасьеву и д.э.н. М.Ю. Головнину за ценные комментарии и замечания, позволившие существенно улучшить работу. Вся ответственность за возможные неточности и опечатки лежит на авторе. 
жетного правила. Абсорбционная способность системы по отношению к государственным расходам отражена в настоящей работе макроэкономической моделью российской экономики, учитывающей структурные особенности системы, основные поведенческие механизмы и механизмы экономической политики.

Как показал вычислительный эксперимент, уровень расходов был занижен по отношению к абсорбционной способности российской экономики. Неоптимальность бюджетной политики является следствием особенностей механизма бюджетного правила, который принципиально исключает возможность выбора текущих расходов бюджета (как инвестиционных, так и на конечное потребление) с учетом их влияния на будущие несырьевые доходы экономики. Оптимальный (более высокий) уровень расходов бюджета расшивает узкие места системы и повышает производственные возможности экономики, делая ее более привлекательной для частных инвестиций. 


\section{1. ОСОБЕННОСТИ БЮДЖЕТНОЙ ПОЛИТИКИ В РОССИИ}

В России было предпринято несколько попыток встраивания стабилизирующего механизма в бюджетную политику. Все попытки представляют собой «нащупывание» долгосрочного уровня нефтегазовых доходов, привязка к которому текущих государственных расходов должна была обеспечить сбалансированность бюджета, сдерживая опережающий (необоснованный) рост расходов, a, кроме того, снизить макроэкономическую волатильность.

С 2000 по 2003 г. расходы федерального бюджета планировались так, чтобы их величина была равна доходам бюджета при цене на нефть 20 долл./барр. - именно этой величине соответствовал средний с 1993 г. уровень цен на нефть (Гурвич, 2010). Определенная таким способом величина, по мнению Правительства, отражала долгосрочный или «нормальный» уровень цен на нефть. Доходы сверх 20 долл. шли исключительно на очередное и досрочное погашение внешнего долга. К положительным результатам снижения уровня долга часто относят ускорение экономического роста, благодаря уменьшению рисков. По расчетам авторов в работе (Гурвич и др., 2010), снижению долга за 2000-2005 гг. соответствует повышение темпов роста на 0,5-1,0\%. По мере сокращения долга ниже безопасного уровня, однако, целесообразность такой формы расходования дополнительных доходов начала ставиться под сомнение как со стороны отраслевых министерств и Министерства экономического развития, так и со стороны экспертного сообщества (Гурвич, 2006).

В период с 2004 по 2007 г., для продолжения прежнего курса политики в условиях растущих нефтяных цен требовалось выработать механизм управления сырьевыми доходами экономики, что привело к созданию Стабилизационного фонда. До 2006 г. в Стабфонд по-прежнему направлялись доходы сверх базовой (нормальной) цены, равной 20 долл. С 2006 г., в результате изменения оценок долгосрочной цены на нефть, базовая цена была повышена до 27 долл. Повышение цены отсечения встречало сопротивление со стороны Министер- 
ства финансов РФ. По мнению Минфина, дополнительные расходы бюджета не оказывали нужного эффекта (для экономического роста), но несли в себе значительные инфляционные риски.

Дальнейшее накопление государственных фондов усилило давление на Минфин. В экспертном сообществе разгорелась дискуссия о наиболее эффективных направлениях расходования средств Стабфонда и соответствующих организационных механизмах. Одна из крайних точек зрения ${ }^{2}$ состояла в признании отсутствия эффективных проектов в экономике - в случае снижения доступности внешнего финансирования неэффективные проекты не смогут обеспечить должной отдачи и целые сектора экономики окажутся на грани дефолта. Наращивание государственных инвестиций, таким образом, рассматривалось как крайне рискованное. Вместо этого Стабфонд предлагалось разбить на три части, каждая из которых выполняла бы отдельную функцию. Гарантийный фонд обеспечивал бы надежность заимствований частного сектора, стабилизационный фонд страховал экономику на случай кризиса, а фонд будущих поколений обеспечивал бы развитие экономики после исчерпания природных ресурсов. Советник Президента А. Илларионов настаивал на недопустимости расходования фонда, «созданного для страховых целей, на любые непроцентные расходы, в том числе социальные и инвестиционные». Расходование средств, по его мнению, возможно лишь за пределами национальной экономики и в первую очередь на цели улучшения имиджа страны - строительство консульств и диппредставительств ${ }^{3}$. Министерство экономического развития, отвечающее за экономический рост, напротив, предлагало перенаправить средства Стабфонда на финансирование инфраструктурных проектов внутри страны. Резкой критике подверглась экономическая политика со стороны Государственной Думы. Так, другая крайняя точка зрения, принадлежащая О. Г. Дмит-

2 Данилов Ю.А., директор Центра развития фондового рынка на круглом столе «Стабилизационный фонд РФ и его роль в экономической политике», организованном Высшей школой экономики в 2005 г.

3 Круглый стол «Стабилизационный фонд: на что и как потратить», организованный Высшей школой экономики в 2004 г. 
риевой, состояла в предложении вовсе отказаться от этого инструмента и направить все средства на социальные расходы, зарплаты и пенсии. Сохранение нефти в месторождениях виделось многим экспертам ${ }^{4}$ более надежной стратегией, чем вывоз капитала в иностранные юрисдикции.

Одним из центральных мест критики стало решение о вложении Стабфонда под 2-3\% годовых в условиях, когда стоимость кредита для частного сектора на международных финансовых рынках составляла 8-15\% годовых. Ставка была сделана на более качественные системы оценки рисков иностранных инвесторов. Кризис конца 2000-х гг., однако, опроверг эту логику - иностранные инвестиции оказались подвержены значительным девальвационным рискам, реализация которых заставила компании отвлекать значительные средства на выплаты внешним кредиторам, отказавшимся рефинансировать долги. Кроме того, доступом к международному рынку капитала в этот период обладали отрасли, имеющие валютную выручку - прежде всего компании сырьевого сектора. Компании внутреннего рынка оказывались в более трудном положении, а банковская система лишалась платежеспособных клиентов, перешедших на внешнее финансирование. Возрастающий спрос на сырье консервировал существующий структурный перекос.

Крайние и близкие к ним точки зрения в экспертном сообществе и Правительстве часто оказывались политизированными. Одна из немногих, лишенных догматизма точка зрения ${ }^{5}$, состояла в том, чтобы «избавиться от представлений об отсутствии экономически целесообразных проектов, которые могли бы быть профинансированы государством» и «делегировать финансовые ресурсы и риски в конкурентную среду», через систему государственных инвестиционных банков, конкурирующих между собой. Подчеркивалось, при этом, что

4 Грачев И.Д., председатель Общероссийского общественного политического движения «Развитие предпринимательства» на круглом столе «Стабилизационный фонд РФ и его роль в экономической политике», организованный Высшей школой экономики в 2005 г. (выступление И.Д. Грачева).

5 Ведев А.Л., Директор аналитической лаборатории «Веди», на круглом столе «Стабилизационный фонд РФ и его роль в экономической политике», организованном Высшей школой экономики в 2005 г. 
проекты должны реализовываться исключительно частным сектором. Предлагалось ${ }^{6}$ также ввести конкуренцию между регионами за государственные ресурсы. Признавалась целесообразность снижения налогового бремени за счет средств Стабфонда в инновационных отраслях экономики, либо в трех - четырех «очевидных» отраслях - локомотивах роста. Так, в докладе ЦМАКП отмечалось, что снижение НДС было бы выгодно для отраслей, ориентированных на внутренний рынок (особенно для машиностроения), в то время как снижение налога на прибыль и единого социального налога стало бы менее эффективным с точки зрения стимулирования экономического роста. В качестве одной из возможных организационных форм государственного инвестирования в этом же докладе предлагается создание Фонда инновационного развития (по аналогии с Фондом Чили), ориентированного на поддержку создания инновационных бизнесов в приоритетных секторах экономики, а также на снижение рисков частных инвесторов, финансирующих инновационные проекты.

В работе (Глазьева, 200677), однако, автор указывает на то, что правительство оказалось неспособным эффективно распорядиться дополнительными доходами, что и привело к проблемам с финансированием: к завышению процентных ставок для предприятий, трудностям в получении кредита. Автор отмечает, также, что проводимая политика оказывала угнетающее действие и на саму банковскую систему, лишая ее ресурсов для развития. Парадокс, по мнению С.Ю. Глазьева, заключался в том, что в качестве главной причины высоких процентных ставок называется высокая инфляции и предлагается еще более масштабное изъятие ликвидности, в то время как истинными причинами являлись: монополизация товаропроводящих сетей, картельный сговор нефтяных компаний и попустительство государства естественным монополиям, в первую очередь ЖКХ, при формировании тарифов. Кредитные возможности, таким об-

6 Яковлев А.А., проректор НИУ ВШЭ, на круглом столе «Стабилизационный фонд РФ и его роль в экономической политике», организованном Высшей школой экономики в 2005 г.

7 http://www.glazev.ru/econom_polit/288/ 
разом, должны быть расширены, что приведет к росту производства и снижению цен.

В докладе ЦМАКП (Белоусов, Солнцев, 2005) отмечается, что накопление поступающих в Стабилизационный фонд средств на счетах в Банке России имеет риск вызвать избыточный стерилизационный эффект и спровоцировать банковский кризис ${ }^{8}$.

В последнее время появился ряд работ, исследующих эффект перераспределения расходов в рамках прежнего объема бюджета. Так, в работе (Кудрин, Кнобель, 2017) исследуется вопрос величины мультипликатора по различным группам расходов. Наряду с квантификацией эффектов бюджетной политик обсуждаются возможности и ограничения бюджетного маневра. Подход на основе анализа мультипликаторов, однако, имеет свои ограничения, связанные с тем, что при вычислении мультипликатора механизмы влияния, присущие российской экономки не прослеживаются.

В работе (Идрисов, Синельников, 2013) обсуждаются теоретические аспекты бюджетной политики в контексте экономического роста, в результате чего авторы приходят к выводу о существование определенного уровня расходов, ниже которого влияние госрасходов на рост положительно, а после достижения этого уровня влияние на рост становится отрицательным. Приводится ряд доводов в пользу отсутствия возможности наращивания государственных расходов в России.

Несмотря на предпринятые правительством попытки формирования системы управления развитием и системы финансирования роста: создание институтов развития, разработки стратегических планов для некоторых отраслей и регионов, внедрение механизмов частно-государственного партнёрства и проектного финансирования, работающей системы создать так и не удалось. Отсутствие единой системы управления ростом не позволило восполнить недо-

8 Некоторые эксперты в качестве основной причины кризиса 2004 г. называют запуск механизма стабилизационного фонда (см., например, Н. Орлова, главный экономист Альфа-банка). 
статок межотраслевой координации, а также наладить координацию разных составляющих экономической политики - бюджетной, монетарной, промышленной (Стратегия модернизации..., 2010). В условиях недостатка координации многие проекты продолжали оставаться высокорискованными, что добавляло аргументов сторонникам сбережения дополнительных доходов бюджета.

Дискуссия о форме бюджетной политики редко доходила до обсуждения конкретных параметров бюджетной системы, а между тем именно обоснованность величины изъятия ликвидности инструментом Стабфонда, а, следовательно, уровень расходов бюджета является одним из ключевых мест.

Размер изъятия тесно связан со спросом на деньги. В работе (Гурвич, 2006), отмечается, что «платой» за стабильность, достигнутую сбережением большой доли конъюнктурных доходов, то есть доходов, образующихся в результате превышения ценой нефти своего нормального уровня, стало сдерживание внутреннего спроса. По мнению автора, однако, в силу близости к нулю в реальном выражении ставок заимствований, расходование конъюнктурных доходов вряд ли заметно увеличило бы инвестиционный спрос, в то время как потребительский спрос и без того рос быстрыми темпами. Автор подчеркивает ограниченность возможностей ЦБ при стабилизации платежного баланса и сглаживании колебаний реального курса. Ограниченность же обусловлена приростом реального спроса на деньги, устанавливающего предел на темпы наращивания денежной базы. Отказ от сглаживания монетарных показателей мерами бюджетной политики (например, инвестирование средств СФ не в иностранные активы, а в российские ценные бумаги) уменьшает, по мнению автора, возможности поддержания стабильного обменного курса без инфляционных последствий. Иными словами, меньшее изъятие ликвидности в Стабфонд в результате более высокого уровня расходов означало бы рост чистых внутренних активов ЦБ и как следствие рост денежной базы. Рост же денежной базы в условиях ограниченности спроса на деньги приводил бы к раскручиванию инфляции. Этой позиции придерживался и Минфин. 
Спрос на деньги формируется под влиянием множества факторов и не всегда может быть описан простыми моделями (количественной теорией денег и т.д.). Денежный рынок связан с другими рынками, в том числе с рынком конечных товаров, изменения на которых порождают многообразие сложных взаимоувязанных динамических эффектов, в результате чего спрос на деньги перестает быть статичной величиной. Более широкие кредитные возможности могут привести к росту производства и увеличению товарной массы и, следовательно, более высокому спросу на деньги в одних условиях, а могут приводить лишь к разгону инфляции, не оказывая влияние на рост - в других. Более общей постановкой в контексте определения величины оптимального изъятия денег в Стабфонд является оценка и учет абсорбционной способности экономики.

\section{2. АБСОРБЦИОННАЯ СПОСОБНОСТЬ РОССИЙСКОЙ ЭКОНОМИКИ}

Изменение величины изъятия означает наращивание или сокращение государственных расходов. Эффективность бюджетных расходов ограничена абсорбционной способностью экономики, которая определяется такими структурными характеристиками, как соотношение торгуемого и неторгуемого (сырьевого - несырьевого) секторов экономики, гибкость рынка труда, качество инфраструктуры, уровень коррупции, отклонением фактического выпуска от потенциального и т.д. Кроме того, абсорбционная способность зависит от бюджетной политики и взаимодействия бюджетной политики с монетарной, которая в свою очередь учитывает спрос на деньги. Форсированное наращивание расходов сверх абсорбционной способности перестает оказывать влияние на экономический рост и приводит лишь к ускорению инфляции и расширению неинвестиционного импорта. Если же абсорбционная способность системы находится выше фактического уровня государственных расходов, то бюджетная политика не реализует весь имеющийся потенциал. 
Бюджетные расходы, однако, обладают масштабирующим эффектом (Collier, 2010) на экономику по сравнению с частными инвестициями. Иными словами, государственные расходы (инвестиции), организованные соответствующим образом, могут, расшивая узкие места системы, масштабировать производственные возможности экономики, делать ее более привлекательной для частных инвестиций, повышая эффективность проектов, реализуемых рыночным сектором экономики. Когда масштабирующий эффект дополнительных бюджетных расходов сильный, меньшему объему изъятия ликвидности в Стабфонд и, как следствие, большему денежному предложению будет соответствовать больший объем производства и больший спрос на деньги. Инфляционного давления в этом случае может не возникать, поскольку бюджетная политика воздействует как на сторону агрегированного спроса, так и на сторону агрегированного предложения. Итоговый результат зависит от соотношения эффектов, порождаемых структурными характеристиками системы, а также от реакции со стороны экономической политики.

Адекватным методом определения оптимальной величины изъятия является задача оптимального управления, где в качестве критерия выбран экономический рост, а управляющей переменной является величина государственных расходов. Критерий контролирует инфляционные эффекты, возникающие в результате достижения границы абсорбционной способности системы:

$$
W=\sum_{t=1}^{T}\left[\left(\frac{y_{t}-y_{t}^{p}\left(g_{t}^{r}\right)}{y_{t}^{p}\left(g_{t}^{r}\right)}\right)^{2}+\lambda\left(\frac{p_{t}^{y}}{p_{t-1}^{y}}-1\right)^{2}\right],
$$

где $y_{t}-$ ВВП в реальном выражении; $p_{t}^{y}-$ ценовой индекс (дефлятор ВВП); $y_{t}^{p}$ - некоторые целевые темпы роста экономики, таргетируемые экономической политикой; $g_{t}^{r}-$ бюджетная политика. Подобная форма критерия является весьма распространённой при анализе экономической политики (Fair, 1984), в особенности при анализе монетарной политики. В контексте настоящего иссле- 
дования в качестве целевого темпа роста используется потенциальный выпуск, задаваемый производственной функций с постоянной эластичностью замещения между трудом и капиталом. Важно, что теперь, при описании потенциального выпуска, допускается влияние на него управляющей переменной политики - государственных расходов. Иными словами, идентифицируется масштабирующий эффект государственных расходов на производственные возможности экономики. Для отражения производственных возможностей экономики оценивается производственная функция и идентифицируется влияние на потенциальный выпуск со стороны государственных расходов (краткое описание используемого подхода дано в Приложении).

Далее, для «вычисления» абсорбционной способности системы используется макроэкономическая модель российской экономики, описание, оценивание и верификация которой содержится в (Скрыпник Д.В. 2016). Модель описывает все релевантные экономические механизмы и позволяет моделировать последствия изменения государственных расходов. Моделируется совокупный спрос (рынок конечного потребления, рынок инвестиционных товаров, внешний спрос) и спрос на деньги, а кроме того, возникающие между двумя рынками связи. В части описания механизмов экономической политики модель учитывает взаимодействие ЦБ и бюджетной системы в контексте накопления ЗВР и бюджетных фондов. Подчеркнем, что в задаче оптимального управления изменение расходов связано с изменением величины изъятия. Рост расходов, таким образом, не предполагает увеличение долгового финансирования и налоговой нагрузки в экономике. При этом рост расходов приводит к сокращению государственных сбережений. Это влияет на объем ЗВР и на объем ликвидности в экономике. Возникающие макроэкономические эффекты предполагают реакцию со стороны ЦБ в соответствии с его предпочтениями в отношении курса, инфляции и роста, что описывается в модели. Учитывается эффект влияния государственного долга на динамику частных инвестиций - так называемый эффект вытеснения, что позволяет описывать динамику системы в услови- 
ях полного исчерпания госфондов. Модель, таким образом, позволяет проследить влияние бюджетной политики на состояние денежного рынка, а также влияние на рынок труда и реальный сектор. Важно, что модель является динамической и позволяет учесть межвременные эффекты. Остальные механизмы и особенности модели подробнее обсуждаются ниже, при анализе результатов вычислений. Общая схема модели представлена на рис. 1.

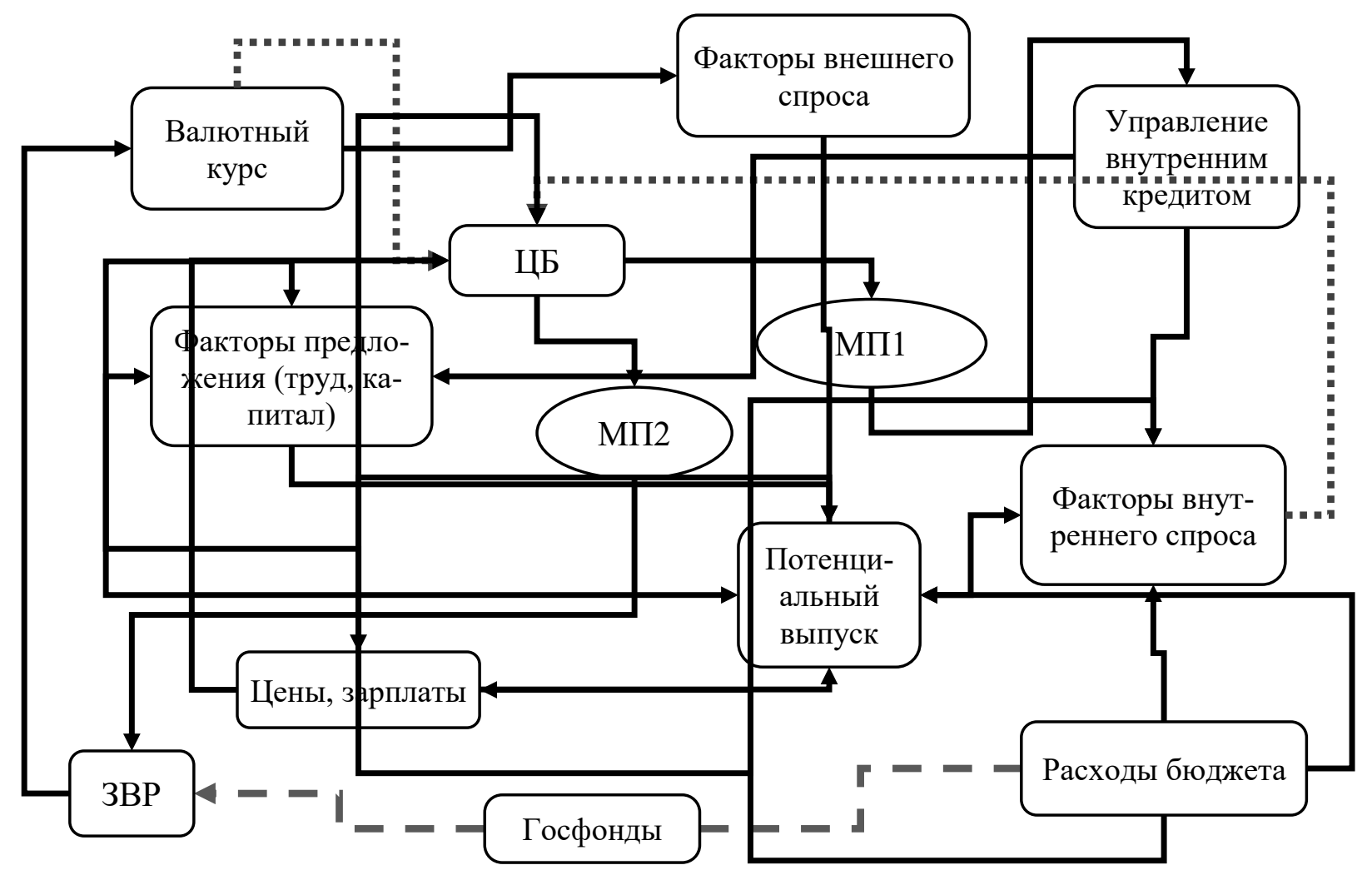

Рис. 1. Общая схема модели*

* МП - монетарное правило. Пунктирной линией выделено влияние целей на решения ЦБ. Длинным пунктиром выделена связь бюджетной системы и монетарной политики.

В общем виде задача оптимального управления записывается следующим образом:

$$
\left\{\begin{array}{l}
W\left(y_{t}, x_{t}, z_{t}\right) \underset{z_{1,} . . z_{T}}{\rightarrow} \min , \\
\text { s.t. } \\
f_{i}\left(y_{t}, x_{t}, z_{t}, a_{i}\right)=u_{i, t}, i=1, \ldots, n,
\end{array}\right.
$$


где $W\left(y_{t}, x_{t}, z_{t}\right)$ - функция критерия, а $f_{i}\left(y_{t}, x_{t}, z_{t}, a_{i}\right)=u_{i, t}$ общая запись макроэкономической модели системы; $y_{t}$ - набор эндогенных переменных; $x_{t}-$ набор экзогенных переменных; $a_{i}$ - параметры модели; $z_{t}$ - переменные экономической политики (в нашем случае - объем государственных расходов).

Реализация поиска оптимального решения осуществляется в соответствии со следующей итерационной схемой. Определяется диапазон изменения значений управляющего параметра. Выбирается шаг изменения управляющего параметра и соответствующее величине шага количество будущих итераций алгоритма. Сам временной интервал разбит на два подинтервала: с 2006 по 2008 гг. и с 2009 по 2011 гг. Управляющий параметр изменяется на каждом подитервале в отдельности. Каждая итерация состоит из трех этапов. На первом этапе на основе эконометрической зависимости вычисляется траектория потенциального выпуска в зависимости от траектории управляющего параметра - государственных расходов - на рассматриваемом временном интервале. Далее, на втором этапе, вычисляются значения эндогенных переменных модели в зависимости от расчётного значения потенциального выпуска и заданной траектории госрасходов (перечень эндогенных переменных дается в Приложении). Кроме того, на этом этапе также на основе модели вычисляется значение совокупного выпуска и инфляции в зависимости от заданной траектории государственных расходов. На третьем этапе на основе полученных траекторий занятости и запаса капитала вычисляется новая траектория потенциального выпуска. Наконец на основе полученной траектории потенциального выпуска в зависимости от расчетной динамики факторов производства, а также с учетом траектории совокупного выпуска и траектории инфляции определяется значение критерия.

После выполнения всех итераций алгоритма выбирается значение управляющего параметра, которому соответствует максимальное значение критерия оптимизации. Отметим, что пересчет траектории потенциального выпуска на третьем этапе каждой итерации алгоритма позволяет наиболее полно 
учесть совокупность трансмиссионных механизмов экономики и обеспечить определенную консервативность критериальной оценки. 


\section{3. РЕЗУЛЬТАТЫ ВЫЧИСЛИТЕЛЬНОГО ЭКСПЕРИМЕНТА}

Средний уровень расходов федерального бюджета в период с начала 2006 по конец 2008 г. составлял порядка 15\% от ВВП. При решении задачи оптимального управления рассматривается диапазон вариации управляющей переменной политики - государственных расходов - от 5 до 30\% ВВП. Управляющая переменная варьируется на двух интервалах: с 2006 по 2008 г. и с 2009 по 2011 г. включительно. При этом критерий вычисляется для совокупного интервала, что позволяет учесть возникающие динамические эффекты бюджетной политики.

Согласно полученному решению задачи ${ }^{9}$, оптимальными значениями расходов федерального бюджета являются уровни 20,95\% ВВП для первого интервала и 20,05\% ВВП для второго. Отметим, что оптимальный уровень для первого интервала выше фактического, а для второго - ниже фактического. Несмотря на некоторое снижение интенсивности бюджетного стимулирования экономики во втором периоде, темпы роста в оптимальном сценарии оказываются выше фактических.

Таблица 1

\section{Teмnы роста ВВП}

\begin{tabular}{l|c|c|c|c|c|c}
\hline & 2006 & 2007 & 2008 & 2009 & 2010 & 2011 \\
\hline Фактический темп & 108,7 & 109,8 & 103,0 & 91,6 & 103,5 & 105,4 \\
Оптимальный темп & 110,3 & 110,6 & 103,7 & 94,2 & 103,7 & 106,7 \\
\hline
\end{tabular}

Рассмотрим основные механизмы возникновения экономического роста при более высоком уровне государственных расходов, обращая при этом внимание на наиболее существенные его отличия от реально складывавшейся ситуации. На представленных ниже графиках результаты, полученные из расчетов

9 Приводятся результаты для значения весового параметра $\lambda$, равного 1. Результаты оказываются устойчивыми к выбору весового параметра. 
по оптимизационной модели, сопоставляются с соответствующими реальными макропоказателями. Сплошные линии отображают фактические данные, пунктирные - решение модели при оптимальном уровне государственных расходов.

Важной особенностью модели является отражение предпочтений ЦБ в отношении инфляционных, курсовых целей и целей роста при управлении денежной базой и ЗВР. Рост расходов федерального бюджета приводит к уменьшению объемов изъятия и росту денежной базы, на что ЦБ реагирует замедлением темпов роста ЗВР (рис. 2). Снижение темпов накопления ЗВР Центральный банк, однако, не делает пропорциональным снижению масштабов изъятия и допускает рост денежной базы (рис. 3), что приводит к расширению кредитных возможностей, но достаточно умеренному, чтобы не спровоцировать вход системы в инфляционную спираль.

Основной механизм возникновения роста связан с расширением производственных возможностей экономики. Государственные расходы, в результате устранения узких мест, увеличивают потенциал системы (масштабируют экономику), делая ее более привлекательной для частных инвестиций. В результате возникает дополнительный инвестиционный спрос со стороны частного сектора и увеличивается накопление капитала, что в свою очередь также увеличивает потенциальный выпуск (рис. 4, 5). Поскольку рост финансируется средствами государственных фондов, а не выпуском государственного долга, денежный рынок не испытывает снижения ликвидности и эффекта вытеснения частных инвестиций не возникает.

Заметим, что в начале первого периода, когда безработица была далека от своего рекордно низкого уровня в предкризисный период, увеличивается спрос на трудовые ресурсы при этом в сам предкризисный период дополнительного давления рынок труда не испытывает (рис. 6). Во втором, послекризисном периоде, безработица во все годы оказывается на более низком уровне по сравнению с фактическим. 
Наблюдается умеренный рост потребления, с некоторым снижением оптимального уровня относительно фактического в послекризисный период, в силу неизбежного в краткосрочном периоде эффекта перераспределения национальных ресурсов (в силу их ограниченности) от потребления к инвестициям для финансирования роста. Тем не менее, по мере раскручивания инвестиционных циклов, потребление в оптимальном сценарии выходит в конце этого периода на более высокий по сравнению с фактическим уровень (рис. 7).

Кроме того, по мере роста производства и соответствующего роста спроса на деньги, ЦБ начинает адекватно наращивать денежную базу. Теперь, в условиях более высокого спроса на деньги, расширяются возможности нейтрализации внешних шоков мерами монетарной политики. Более того, меняется канал предоставления ликвидности. Если раньше основным был канал скупки валюты и валютный рынок, то теперь основными становятся канал банковского кредитования и канал госрасходов. Происходит раскручивание спирали роста, и экономика выходит на более эффективный режим. Возросшая инвестиционная привлекательность экономики в конце периода обуславливает более высокий объем частных инвестиций, даже несмотря на более низкий объем государственных расходов по сравнению с фактическим в послекризисный период (рис. 4).

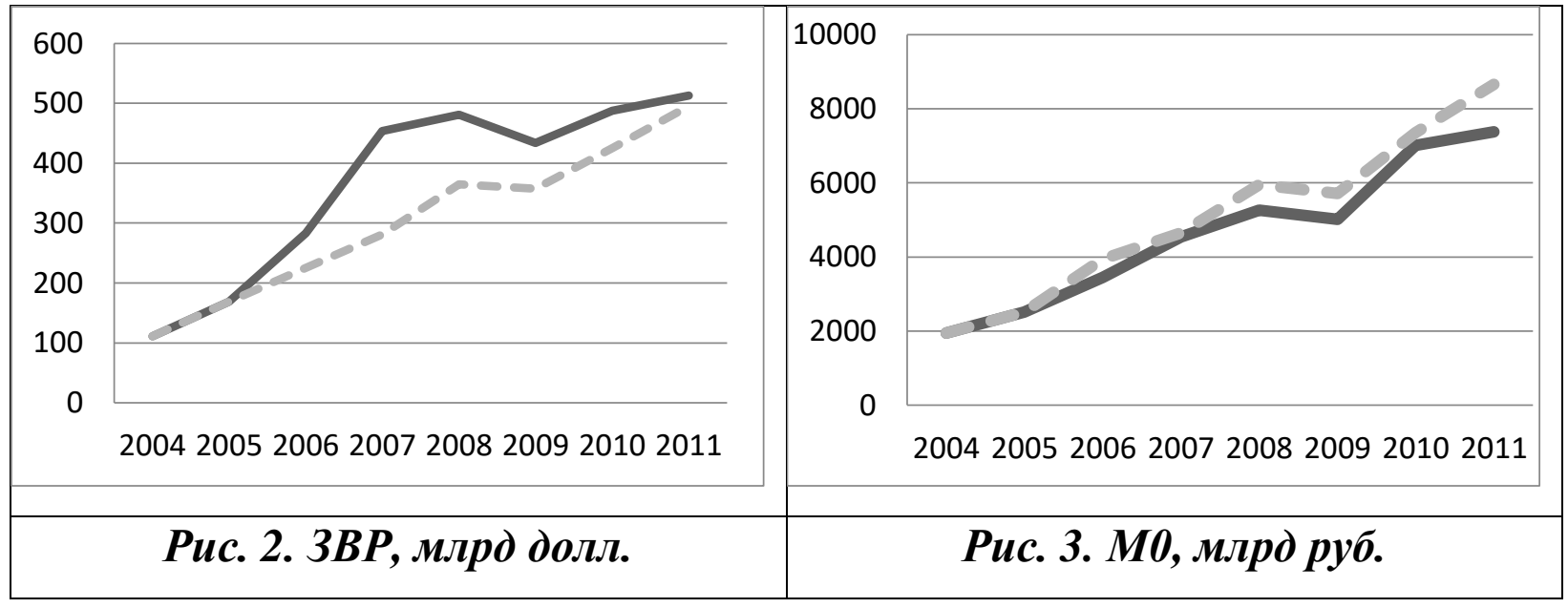




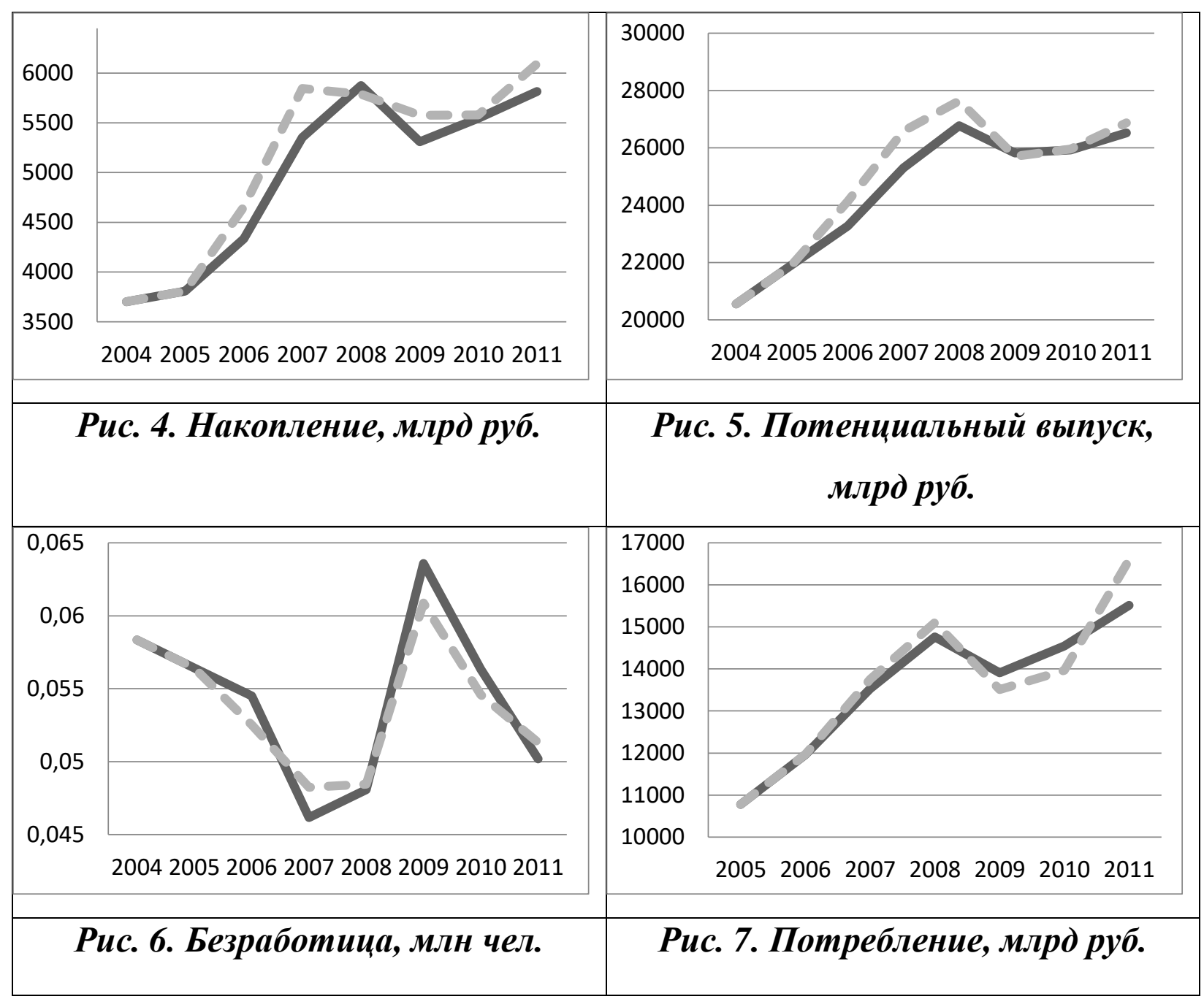

Рассмотрим далее внешний сектор и курсовую динамику. Сокращение темпов накопления ЗВР приводит к укреплению валютного курса рубля в самом начале интервала, как номинального (рис. 8), так и реального (рис. 9). Следствием укрепления рубля является сокращение ненефтяного экспорта и увеличение импорта ${ }^{10}$, сокращается чистый экспорт и, как результат, приток валюты. Снижение притока валюты приводит к ослаблению номинального курса рубля и, в условиях большей ценовой инерционности, ослаблению реального курса рубля ${ }^{11}$. В таких условиях удерживать курс рубля от номинального и реального укрепления центральному банку в соответствие со своим правилом удается меньшим темпом накопления ЗВР. Рост производительности труда в

${ }^{10}$ Рост показателя связан также с тем, что часть государственного спроса и частного инвестиционного спроса покрывается импортом.

${ }^{11}$ Реальному ослаблению рубля в оптимальном сценарии также способствует рост выпуска в неторгуемом секторе экономике и, как следствие, снижение цены неторгуемых товаров, на которые приходится основной прирост государственных расходов в оптимальном сценарии по сравнению с фактическим объемом расходов. 
первом периоде в результате расширения производственных возможностей экономики находит отражение в росте ненефтяного экспорта во втором периоде по отношению к фактическим значениям (рис. 10). Отметим, что динамика нефтяного экспорта (рис. 12) остается практически неизменной, что вполне ожидаемо.

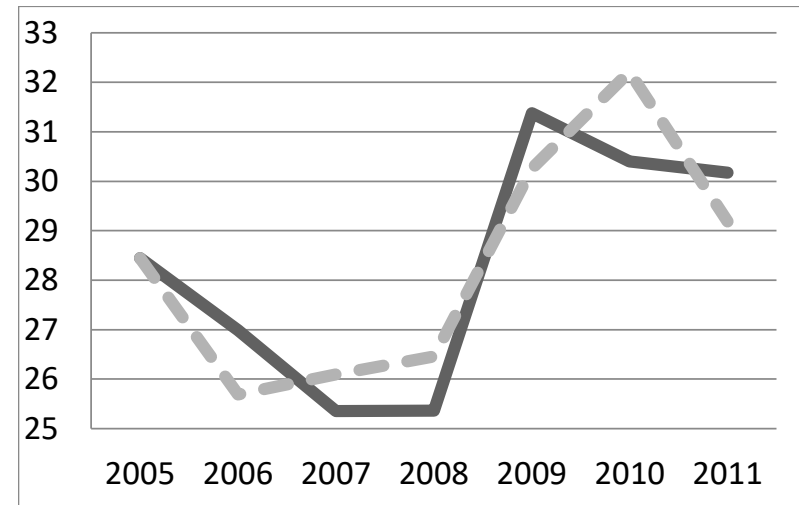

Рис. 8. Курс доллара, руб.

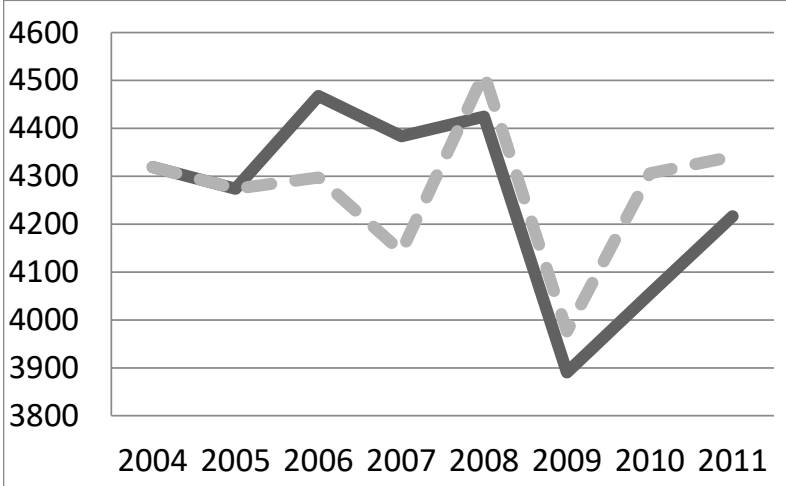

Рис. 10. Ненефтяной экспорт, млрд руб.

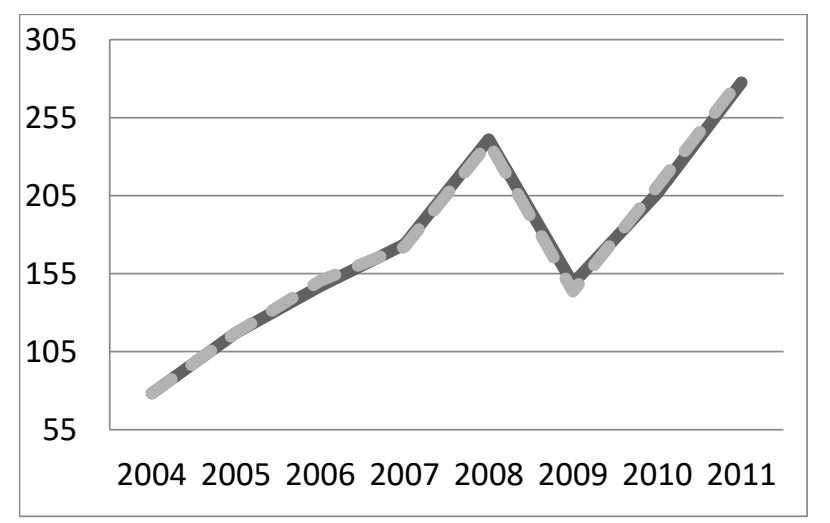

12 Рост индекса означает укрепление реального валютного курса рубля.

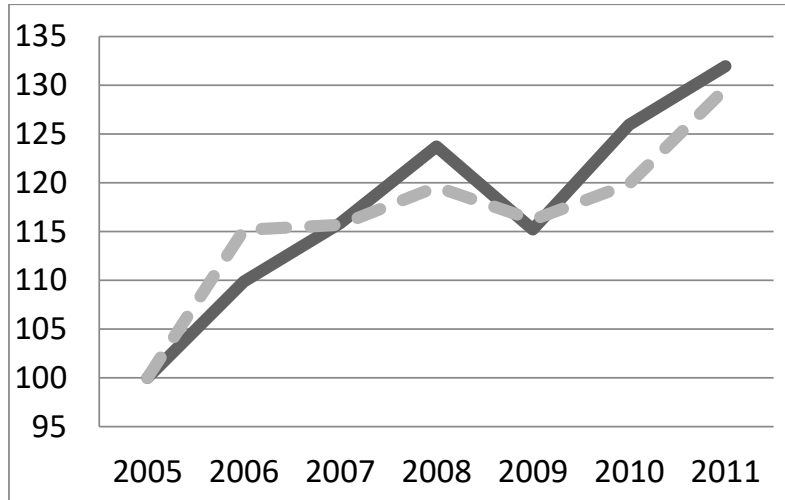

Рис. 9. Индекс ${ }^{12}$ реального курса рубля, \% (2005 г. = 100\%)

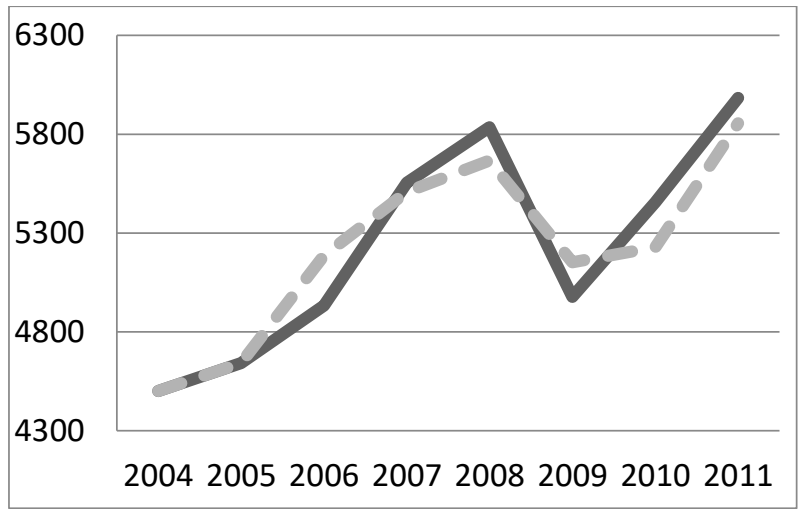

Рис. 11. Импорт, млрд руб.

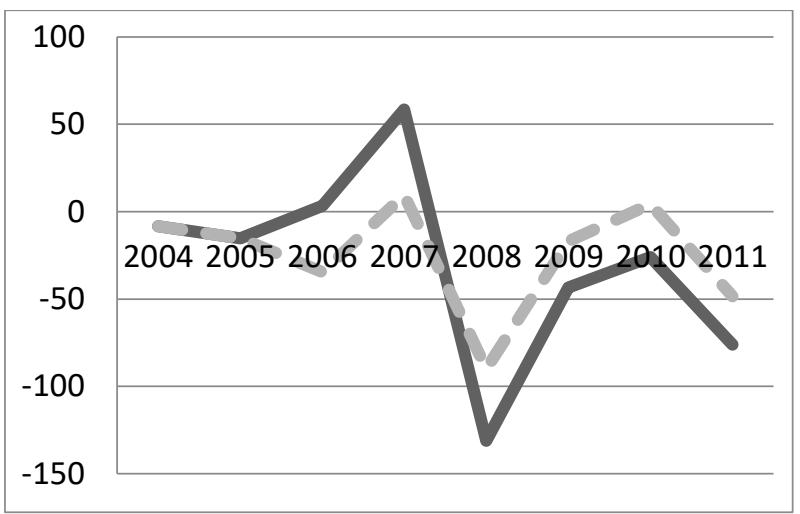




\section{Рис. 12. Нефтяной экспорт, млрд Рис. 13. Приток частного капитала,}

долл. млрд долл.

Рассмотрим ценовую динамику. Как можно заметить (рис. 14 и 15), в первой половине оптимального сценария возникает инфляционное давление в результате опережения роста совокупного предложения ростом совокупного спроса. В результате ускорения экономического роста, однако, затем возникает обратный эффект: во второй половине интервала ИПЦ в оптимальном сценарии оказывается ниже фактических значений. Следует подчеркнуть, что снижение инфляции происходит в условиях прежнего курса политики ЦБ (модель оценивалась на интервале до 2011 г.), а не в результате интенсификации перехода к режиму инфляционного таргетирования в более поздний период.

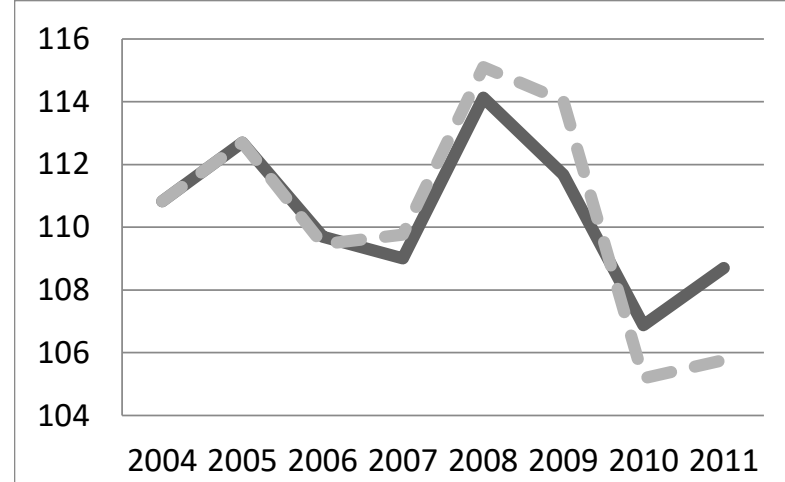

Рис. 14. ИПЦ, год к году, \%

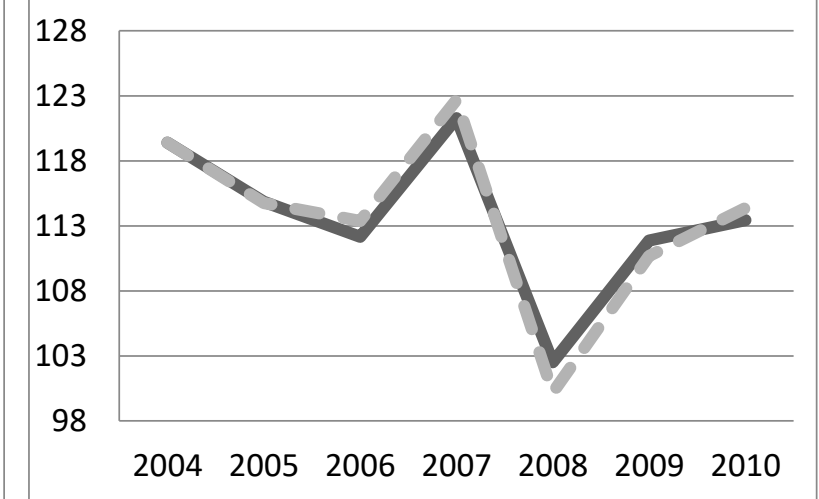

Рис. 15. Дефлятор ВВП, год к году, \%

Наконец, более высокие темпы роста экономики увеличивают доходы бюджета. На рис. 16 в докризисном периоде после скачка вначале интервала заметен тренд на возрастание профицита. В послекризисном периоде, также после скачка вначале интервала, заметен тренд на сокращение дефицита бюджета. В оптимальном сценарии, таким образом, достигается сбалансированность бюджета, но не в результате сдерживания расходов бюджета, а в результате роста экономики и более высоких несырьевых доходов бюджета. 


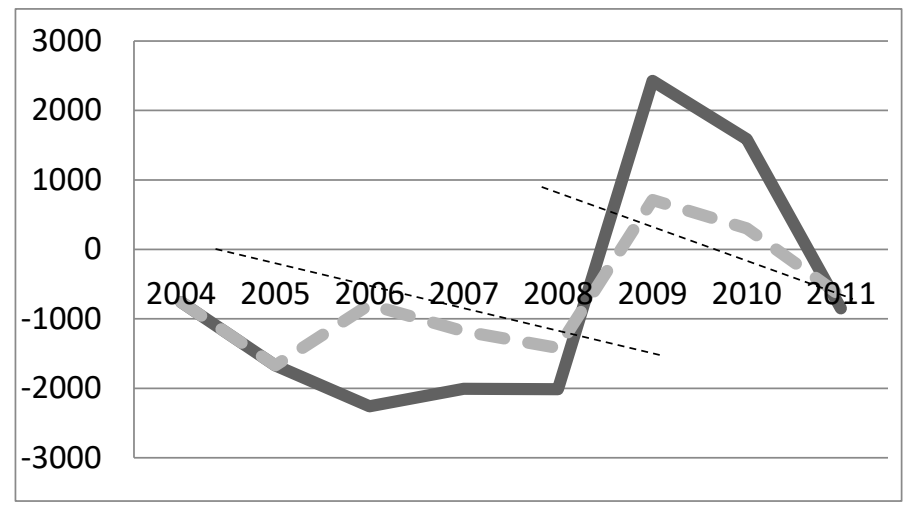

Рис. 16. Профицит (дефицит), млрд руб.

Результаты вычислительного эксперимента, таким образом, позволяют сделать следующие выводы.

- Абсорбционная способность экономики допускала наращивание инвестиционных расходов и расходов на конечное потребление государства. Усилия же по их сдерживанию оказались чрезмерными. Бюджетная политика, таким образом, обладала нереализованным потенциалом роста.

- Основным фактором роста в оптимальном сценарии является масштабирующий эффект госрасходов. Перегрева экономики не происходит, поскольку экономическая политика воздействует сразу на две стороны - спроса и предложения.

- Долгосрочная сбалансированность бюджета могла быть достигнута на основе роста несырьевых доходов - более высокий их уровень увеличивает «безопасный» порог госрасходов.

- Более широкие кредитные возможности экономики в оптимальном сценарии обеспечили бы «мягкое» падение экономики в период кризиса 2008 2009 гг. 


\section{4. ОПТИМАЛЬНОЕ БЮДЖЕТНОЕ ПРАВИЛО}

В России на протяжении 2000-х гг. Правительство фактически использовало бюджетное правило структурного баланса, согласно которому величина структурных доходов экономики и доходов бюджета определяется в зависимости от цены нефти («нормальной» или долгосрочной). Величиной структурных доходов, при этом, ограничивались как расходы на конечное потребление, так и инвестиционные расходы государства.

Механизм бюджетного правила принципиально исключает возможность выбора текущих расходов бюджета (как инвестиционных, так и на конечное потребление) с учетом их влияния на будущие несырьевые доходы экономики. Бюджетной политике, таким образом, отводится ведомая роль - она обязана следовать за конъюнктурой внешнего рынка. В терминах управления действующий в России тип правила относится к задаче управления по разомкнутому контуру, в то время как правило, учитывающее влияние расходов на рост относится к задаче управления по замкнутому контуру: расходы - рост - расходы. Как показал проведенный вычислительный эксперимент, правило, основанное на управлении по разомкнутому контуру, не позволяет реализовать оптимальную для России бюджетную политику.

Одной из основных целей правила структурного баланса является снижение процикличности бюджетной политики, что как раз и достигается разрывом контура рост - расходы. С одной стороны, это исключает выбор расходов с учетом их влияния на рост, но с другой позволяет избежать переноса шоков на бюджетную политику и снизить тем самым процикличность. Фокус политики на снижении процикличности в развитых странах оправдан, поскольку вклад бюджетной политики в рост становится все менее значимым по сравнению с вкладом рыночного сектора экономики. В этом случае положительный эффект для роста от стабилизации оказывается большим по сравнению с эффектом стимулирующей бюджетной политики. Правила подобной структуры подходят 
для развитых стран, богатых ресурсами, таких как Канада, Норвегия, Австралия, не испытывающих недостатка инфраструктурного и производительного капитала, находящихся вблизи или на стационарной траектории сбалансированного роста - когда рыночное взаимодействие обеспечивает оптимальное распределение ресурсов, а шоки приводят к колебаниям вокруг стабильного тренда ${ }^{13}$. Использование правил, не включающих в свою структуру механизм управления по замкнутому контуру, развивающимися странами, и в частности Россией, может оказаться препятствием для решения целого комплекса вопросов экономического развития, многие из которых не имеют стандартного решения, а вопрос стабилизации государственных расходов является лишь одним из многих.

Рыночное взаимодействие в развивающихся странах зачастую оказывается не способным обеспечить оптимальное для устойчивого экономического развития макроструктурные, отраслевые пропорции. Бюджетная же политика, основанная на бюджетном правиле с управлением по замкнутому контуру, могла бы таргетировать оптимальные макроструктурные пропорции напрямую. В условиях отсутствия эффективной координации рыночными (ценовыми) механизмами, многие эффективные проекты «не видны» на уровне отдельной отрасли либо остаются высокорискованными. Слабость системы выбора проектов либо ее отсутствие является одной из основных причин низкой эффективности бюджетных инвестиций. Институционализация управления по замкнутому контуру позволит восполнить недостаток межотраслевой координации. Активное бюджетное правило сделает необходимым согласование краткосрочных целей с долгосрочными. «Маршрут» достижения целей позволит выявить «узкие» места в экономике, ее структурные ограничения, а также выявить принципиально неосуществимые в текущем состоянии системы проекты. Одним из возможных

13 Кроме того, логика простых правил структурного баланса исключает возможность учитывать в той или иной форме предпочтения экономических агентов. Известно, что богатые потребители ценят дополнительную единицу потребления намного меньше, чем эту же единицу ценят бедные потребители. Следовательно, перераспределяя потребление от будущих более богатых поколений к нынешнему более бедному поколению, можно повысить интегральную полезность. 
мест приложения усилий мог бы стать неторгуемый сектор экономики. Устранение «узких» мест в этом секторе позволит повысить абсорбционную способность системы, а рост производительности труда ослабит негативные последствия для курсовой динамики в условиях более низких темпов накопления ЗВР. Рост производительности труда в неторгуемом секторе экономики, наряду с накоплением ЗВР, является одним из рецептов лечения голландской болезни (Berg, 2011).

Формально бюджетное правило, основанное на управлении по замкнутому контуру, может быть представлено решением оптимизационной задачи (5), в которой функция критерия отражает цели бюджетной политики на планируемый период, такие как экономический рост, снижение инфляции, макрофинансовая стабильность и т.д. Важным аспектом предложенной схемы является повышение качества прогнозирования экзогенных для российской экономики переменных, в том числе и с привлечением ведущих научных организаций.

Институционализация бюджетного правила с управлением по замкнутому контуру предполагает его интеграцию с системой выбора проектов. В мировой практике сложилось два основных типа систем управления ростом (Полтерович, 2015). Первый тип - универсальное планирование, ориентированное преимущественно на догоняющее развитие. Этот тип подходит для сильно отстающих стран. Второй тип - это программное планирование, реализуемое бюджетными программами. Планирование этого типа реализуют в основном развитые страны. В России создавались элементы как универсального, так и программного планирования, но единой системы управления ростом создать так и не удалось. В чистом виде ни один из типов планирования не подходит для страны, находящейся на том уровне развития, на котором находится российская экономика. Бюджетное правило с элементами управления по замкнутому контуру, способное генерировать оптимальную бюджетную политику, сопряженное с системой выбора проектов, может стать основой формирования 
институционального механизма, основанного на сочетании систем планирования двух типов.

Включение в реальный процесс бюджетного планирования механизм управления по замкнутому контуру является непростой задачей. Реализация такого механизма потребует существенного изменения сложившейся системы государственного управления, межведомственного взаимодействия в процессе бюджетного планирования. Потребуется создание надежных методов оценивания, отбора крупномасштабных проектов, формирование на их основе эффективного портфеля, а также методов управления реализацией проектов. В частности, потребуется разработка больших структурных межотраслевых моделей, позволяющих описывать количественно макроэкономические последствия качественных изменений: способов и издержек производства существующей продукции, внедрение новых продуктов и т.д.. В окончательном варианте система должна уже на стадии подготовки бюджета выбирать оптимальную конфигурацию (с точки зрения достижения цели роста, дополнительных расходов, инфляционных и других макроэкономических эффектов, а также межвременных эффектов) портфеля проектов, соответствующий ей объем дополнительного финансирования и предельный уровень ограничения правила, соответствующий, таким образом, абсорбционной способности экономики.

В реальной практике бюджетного планирования надежный расчет множества проектов и вариантов конфигурации портфеля потребует существенных усилий, а, значит, не сможет исключить вероятность принятия неэффективных решений по инвестированию и расходованию бюджетных средств, по крайней мере, на стадии создания системы. Для минимизации рисков принятия неверных решений и сохранения принципа разумной консервативности бюджетной политики, целесообразным представляется использование скользящего механизма обратной связи. Речь идет о поэтапном снижении или увеличении границы расходов бюджета на величину дополнительных ненефтегазовых доходов бюджета, возникающих от реализации портфеля проектов. Если фактические 
дополнительные доходы соответствуют запланированным, то это означает, что абсорбционная способность экономики не достигнута и, следовательно, возможно дальнейшее снижения ограничения правила (увеличения расходов бюджета) на предстоящий год либо трехлетний плановый период. Наоборот, если ненефтегазовые доходы бюджета оказываются ниже запланированных, то это указывает на достижение предела абсорбционной способности и является основанием для поднятия границы расходов (снижение расходов) на предстоящий период. Таким образом, происходит подстройка бюджета к структурным возможностям экономики, одновременно ослабляются, что важно, без потери управляемости бюджетного процесса, ограничения роста экономики. Процесс фактически повторяет механизм нащупывания равновесия, лежащий в основе функционирования реального рынка. Подобный механизм обеспечивает включение принципа проектного финансирования - когда финансирование проекта осуществляется преимущественно доходами от самого проекта - в бюджетный процесс. Внедрение механизма представляется целесообразным в свете инициатив Правительства РФ по созданию «фабрики проектного финансирования» на базе Внешэкономбанка.

Предлагаемый механизм сможет обеспечить достаточную степень прогрессивности бюджетной политики, став, при этом, частью общей конструкции бюджетного правила. Функция изоляции бюджета от волатильности цен на нефть за бюджетным правилом должна сохраняться. 


\section{ЗАКЛЮЧЕНИЕ}

Проводимая в период 2000-х гг. в России политика по управлению сверхдоходами от продажи углеводородов часто становилась предметом споров и дискуссий. Действительно, если форсированное наращивание расходов превосходит абсорбционную способность экономики, то это не приведет к росту экономики, а вызовет лишь рост цен. Как показал, однако, вычислительный эксперимент, абсорбционная способность российской экономики по отношению к государственным расходам не была полностью востребована, а имеющийся потенциал бюджетной политики остался не реализованным.

Бюджетные расходы, организованные соответствующим образом, расшивая узкие места системы, масштабируют производственные возможности экономики, делая ее более привлекательной для частных инвестиций, повышая эффективность проектов, реализуемых рыночным сектором экономики. Адекватное приросту спроса на деньги наращивание предложения денег позволяет не допустить раскручивания инфляционной спирали. Перегрева системы не происходит, поскольку экономическая политика воздействует сразу на две стороны - спроса и предложения.

Наблюдаемое в оптимальном сценарии в начале периода некоторое сжатие чистого ненефтяного экспорта приводит к сокращению притока валюты. В условиях меньшего притока валюты меньшего темпа роста ЗВР становится достаточно для сглаживания колебаний реального курса. Но уже в следующий период, вслед за ростом потенциала экономики, расширяется чистый ненефтяной экспорт и возрастает темп накопления резервов.

Механизм действующего в России бюджетного правила принципиально исключает возможность выбора текущих расходов бюджета (как инвестиционных, так и на конечное потребление) с учетом их влияния на будущие ненефтяные доходы экономики. Этот тип правила представляет собой механизм управления по разомкнутому контуру и подходит только для развитых стран. Для 
развивающихся же стран, конструкция должна включать замкнутый управленческий контур, сопряженный с системой выбора проектов. 


\section{ПРИЛОЖЕНИЕ}

Эндогенные переменные модели. Реальный блок: ВВП, потребление, склонность к потреблению, инвестиции в основной капитал, запас капитала, приток частного иностранного капитала, нефтегазовый экспорт, ненефтегазовый экспорт, импорт. Рынок труда: безработица, зарплата. Монетарный блок: золотовалютные резервы, денежная база, денежный мультипликатор, денежный агрегат М2. Бюджетный блок: доходы бюджета (НДС, НП, импортные, экспортные пошлины, НДФЛ и др.), расходы бюджета. Цены: дефлятор российского ВВП, российский индекс потребительских, цен, обменный курс доллара к рублю, реальный эффективный курс рубля

Экзогенные переменные модели: цена нефти, импорт стран ЕС, темпы роста мировой экономики, дефлятор ВВП США, дефлятор ВВП ЕС, ИПЦ США, ИПЦ ЕС, обменный курс доллара к евро, население.

Потенциальный выпуск описывается CES функцией:

$$
y_{t}=\gamma\left(\delta k_{t}^{-\rho}+(1-\delta) e_{t}^{-\rho}\right)^{-1 / \rho},
$$

где $k_{t}$ - запас капитала в момент времени $t ; e$ - объем труда; $\rho$ - эластичность замещения труда и капитала; $\gamma, \delta$ - параметры. Схема идентификации параметров следующая. Параметр $\rho$ идентифицируется на основе коинтегрирующей комбинации уравнения оптимальности для спроса на труд ${ }^{14}$. Коэффициент $\delta$ калибруется на основе средней доли доходов на капитал за период с 2000 по 2011 г. Параметр $\gamma$ и начальный запас капитала, $k_{0}$ идентифицируется эконометрически на основе обобщенного метода моментов. В качестве инструментов мы используем приток капитала, инвестиции и цены на нефть в текущий и прошлые моменты времени. В результате базовым соотношением модели становится уравнение:

$$
y_{t}=175,91 \cdot\left(0,36 \cdot\left(k_{t}+63266\right)^{-0,61}+0,47 \cdot e_{t}^{-0,61}\right)^{-1 / 0,61} .
$$

\footnotetext{
${ }^{14}$ Подробнее см. Скрыпник Д.В., 2016
} 
Для определения влияния со стороны бюджетной политики на потенциальный выпуск идентифицируются структурные шоки бюджетной политики на основе подхода, предложенного в работе (Blanchard, Perotti, 1999). Смысл подхода состоит в разделении бюджетного показателя на инерционную и неинерционнную компоненту. Последняя представляет собой фактически импульсы бюджетной политики или саму политику. Процедура решает проблему эндогенности, возникающую в обычной регрессии роста на госрасходы. Далее, после того как все параметры производственной функции идентифицированы, на основе реальных данных по труду и инвестициям строится оценка динамики потенциального выпуска экономики и идентифицируется влияние на потенциальный выпуск со стороны импульсов бюджетной политики. 


\section{СПИСОК ЛИТЕРАТУРЫ}

Гурвич E.T. Бюджетная и монетарная политика в условиях нестабильной внешней конъюнктуры // Вопросы экономики. 2006. № 3.

Гурвич E.T., Вакуленко Е., Кривенко П. Циклические свойства бюджетной политики в нефтедобывающих странах // Вопросы экономики. 2009. № 2.

Гурвич E.T. и др. Оценка вклада эффекта Балассы-Самуэльсона в динамику реального обменного курса рубля // Вопросы экономики. 2008. № 7. С. 12-30.

Идрисов Г.И., Синельников-Мурылев С.Г. Бюджетная политика и экономический рост //Вопросы экономики. - 2013. - Т. 8. - С. 35-59.

Кудрин А.Л., Кнобель А.Ю. Бюджетная политика как источник экономического роста //Вопросы экономики. - 2017. - №. 10. - С. 5-26.

Полтерович В.М. О формировании системы национального планирования в России // Журнал Новой экономической ассоциации. 2015. № 2 (26).

Скрыпник Д. В. Макроэкономическая модель российской экономики //Журнал Экономика и математические методы (ЭММ). - 2016. - Т. 52. - №. 3. - С. 92-113.

Солнцев О. Г., Белоусов Д. Р. Об использовании ресурсов стабилизационного фонда для стимулирования экономического роста // Проблемы прогнозирования. 2005. №. 4.

Стратегия модернизации российской экономики / Под ред. В.М. Полтеровича. СПб.: Алетейя, 2010.

Berg A. et al. Public investment in resource abundant low-income countries // IMF/CBRT Conference on Policy Responses to Commodity Price Movements, Istanbul, 2012.

Blanchard O., Perotti R. An empirical characterization of the dynamic effects of changes in government spending and taxes on output. № w7269. National bureau of economic research, 1999.

Cherif R., Hasanov F. Oil exporters' dilemma: How much to save and how much to invest // World Development. 2013. T. 52. C. 120-131.

Collier $P$. Savings and investment decisions in low-income resource-rich countries // Centre for the Study of African Economies, Department of Economics. Oxford University, 2011.

Collier P., van der Ploeg R., Spence M., Venables A. Managing Resource Revenues in Developing Economies // IMF Staff Papers. 2010. Vol. 57.1. P. 84-118.

Fair R.C. Specification, estimation, and analysis of macroeconometric models. Harvard University Press, 1984. 


\section{REFERENCES}

Gurvich E.T. Fiscal and monetary policy in an unstable external environment // Voprosy ekonomiki. 2006. № 3 .

Gurvich E., Vakulenko E., Krivenko P. Cyclical Properties of Fiscal Policy in OilProducing Countries // Voprosy ekonomiki. 2009. № 2.

Gurvich E., Sokolov V., Ulyukaev A. The Impact of the Balassa-Samuelson Effect on the Real Ruble Exchange Rate: The Assessment // Voprosy ekonomiki. 2008. № 7.

Idrisov G.I., Sinelnikov-Murylev S.G. Budget Policy and Economic Growth // Voprosy ekonomiki. 2013. № 8 .

Kudrin A.L., Knobel A.Y Fiscal policy as a source of economic growth// Voprosy ekonomiki. 2010. № 10.

Polterovich V. On the Formation of National Planning System in Russia // Journal of the New economic association, 2015, 2(26)

Polterovich V., et al Strategy of Modernization of the Russian Economy // Aleteya, Moscow, 2010

Skripnik D. A Macroeconomic Model of the Russian Economy // Economics and the Mathematical Methods. 2016, № 3

Solntsev, O., and Belousov, D., The Use of Stabilization Fund's Resources for Economic Growth Stimulating // problemy prognozirovaniya, 2005. № 4

Berg A. et al. Public investment in resource abundant low-income countries // IMF/CBRT Conference on Policy Responses to Commodity Price Movements, Istanbul, 2012.

Blanchard O., Perotti R. An empirical characterization of the dynamic effects of changes in government spending and taxes on output. № w7269. National bureau of economic research, 1999.

Cherif R., Hasanov F. Oil exporters' dilemma: How much to save and how much to invest // World Development. 2013. T. 52. C. 120-131.

Collier $P$. Savings and investment decisions in low-income resource-rich countries // Centre for the Study of African Economies, Department of Economics. Oxford University, 2011.

Collier P., van der Ploeg R., Spence M., Venables A. Managing Resource Revenues in Developing Economies // IMF Staff Papers. 2010. Vol. 57.1. P. 84-118.

Fair R.C. Specification, estimation, and analysis of macroeconometric models. Harvard University Press, 1984. 